\title{
Rotation-induced taste aversions in strains of rats selectively bred for strong or weak acquisition of drug-induced taste aversions
}

\author{
RALPH L. ELKINS and WILLIAM HARRISON \\ Veterans Administration Medical Center, Augusta, Georgia 30910
}

\begin{abstract}
Sprague-Dawley-derived rats have been selectively bred as strong or weak learners of a cyclophosphamide-induced saccharin aversion. Resultant aversion acquisition appears to be under strong genetic control, but specific mechanisms of strain differentiation remain to be determined. Current research involves possible generality of strain separation across different modes of aversion induction. Naive offepring of strong and weak-conditioner parents of the F-6 selected generation were used to pair the normal CS saccharin solution with two different magnitudes of an alternate mode of aversion induction, rotational stimulation. Six minutes of intermittent rotation produced no evidence of conditioned aversions within either strain, and equivalent results were obtained from weak conditioners exposed to $10 \mathrm{~min}$ of rotation. However, strong conditioners exposed to $10 \mathrm{~min}$ of rotation acquired significant aversions, thereby demonstrating that strain separation is not simply a function of differential sonditivity to cyclophosphamide, the US basis of genetic selection.
\end{abstract}

Illness-induced taste aversions (TAs) are readily acquired by a variety of species, including rats and humans, when the ingestion of a novel flavor is followed by food poisoning or by either radiation- or druginduced illness (cf. Riley \& Clarke, 1977). TA conditioning methodology and the results of many animal experiments are of considerable applied importance because they directly impact upon TA approaches to alcoholism treatment (e.g., Elkins, 1975, 1980b; Elkins \& Hobbs, 1979). TA learning is a very efficient adaptation, as evidenced by aversion formation following very low doses of illness-inducing drugs, by successful conditioning with long delays between CS and US presentation, and by the strong resistance of acquired aversions to degradation through the processes of extinction and forgetting (cf. Elkins, 1980b). However, despite the robust nature of TA conditioning, marked individual variability in aversion acquisition has been observed both in rats (Elkins, 1973) and in human alcoholics whose treatment included actual or imaginary pairings of alcoholic beverages with drug- or verbally induced nausea [cf. emetic therapy, Voegtlin (1947); covert sensitization (verbal aversion) therapy, Elkins (1980b)]. Voegtlin (1947) reported that a minority of treated alcoholics displayed typical emetic reactions when injected with nausea-inducing drugs but failed to acquire the normal signs of conditioning that generally developed following repeated alcohol-illness pairings. Vogel (1960) suggested that individual differences in autonomic conditioning were responsible for Voegtlin's conditioning failures. Elkins (1980a) observed similar differential conditioning

This work was supported by the Medical Research Service of the Veterans Administration. while studying covert sensitization alcoholism treatment. During covert sensitization, imaginary drinking scenes are repeatedly paired with verbally induced nausea. Most alcoholics who generated verbally induced nausea, as indicated by behavioral observations, physiological recordings, and subjects' self-reports, subsequently developed strong conditioned aversions. However, like Voegtlin's emetic therapy patients, a minority of the covert sensitization subjects failed to show evidence of conditioning despite many apparently successful pairings of drinking scenes with intense nausea. The clinical importance in these differences in conditionability is emphasized by results of follow-up. The conditioning failures reverted to alcohol consumption at a rate that was statistically equivalent to the performance of treatment dropouts (mean $=4.5$ vs. 5.2 months, respectively), but successfully conditioned patients remained abstinent for a therapeutically and statistically significant average of 13.7 months. Clarification of the determinants of these individual differences in conditionability is of obvious scientific interest and could facilitate the development of criteria for selecting alcoholics likely to benefit from TA approaches to treatment. Attempts to separate successful from unsuccessful conditioners on the basis of standard psychological tests have not yet produced significant results, but considerable progress is being made toward the development of an animal model that may be useful in clarification of biological determinants.

In an attempt to develop an animal model suitable for studies of physiological bases of individual differences in conditionability, rats have been selectively bred to be strong or weak learners of conditioned TAs. Sprague-Dawley-derived rats were injected with cyclophosphamide after they consumed a novel saccharin 
solution. The males and females displaying the strongest aversions, as assessed by standard two-bottle preference tests, were mated, and inefficient learners were similarly paired to produce the F-1 generation. Selection has now progressed through the F-7 generation. TA learning appears to be under strong genetic control, as evidenced by progressive strain separation and significant strain differences in TA magnitude ( $\mathrm{p}<.05$ or better) for the F-2 through the F-7 generations (Elkins, Note 1).

Observed strain divergence is consistent with suggestions that efficiency of aversion acquisition is a function of genetically mediated individual differences in TA proneness. However, although some genetic contribution to TA proneness has been demonstrated, the biological bases of strain differences remain to be determined. For example, possible contributions to TA proneness include differential reactivity to the illnessinducing drug, differential sensitivity to the taste of the CS solution, or differences in efficiency of central associative mechanisms underlying aversion formation. The current problem concerns an investigation of the generality of strain separation across different modes of illness induction. Green and Rachlin (1973), as well as Haroutunian and Riccio (1975), following parametric studies of motion sickness, concluded that rotation shared common effects with poisoning and radiation, traditional sources of TA induction. This is a study of the degree to which strain differences can be observed when the CS saccharin solution is paired with rotational stimulation delivered at parametric values judged likely to produce motion sickness.

\section{METHOD}

Following breeding for the F-6 generation, breeders were re-paired with their original partners to produce additional study subjects. Subjects of the present experiment were experimentally naive 90 - to 120-day-old offspring ( 20 males, 20 females) of this rebreeding and were randomly selected from within the strong and weak conditioning strains. Subjects were individually housed in standard wire cages and, except as noted below, had ad-lib access to water and to Purina Laboratory Rat Chow. They were maintained at $72^{\circ} \mathrm{F} \pm 3^{\circ} \mathrm{F}$ under a $0600 \mathrm{~h}$ onset and $1800 \mathrm{~h}$ offset EST light-dark cycle.

Nonconditioned strong and weak TA learners, when tested with the standard two-bottle preference procedure to be described below, have consistently displayed equivalent and high preferences for the $.1 \% \mathrm{CS}$ saccharin solution used during strain selection. It is also known from pilot studies that simple confinement in the rotational apparatus following saccharin ingestion does not reduce subsequent saccharin preference. However, rotational parameters for effective aversion induction within the selected strains were unknown. It was therefore decided that an efficient use of available subjects would involve strain comparisons of the effects of two different amounts of rotational stimulation. Strong conditioners were therefore randomly subdivided into two 10 -member groups, with each group containing 5 male and 5 female subjects. Weak conditioners were similarly subdivided. Conditioning procedures were completed between 1200 and $1600 \mathrm{~h}$. Twenty-six-hour fluid-deprived and 4-h food-deprived subjects were given home cage access to a .1\% solution of sodium saccharin in tap water. Saccharin was avail- able for at least $10 \mathrm{~min}$, for a minimum of $5 \mathrm{~min}$ following the onset of drinking, and for a maximum of $30 \mathrm{~min}$. Five minutes after the end of saccharin availability, each subject was removed from the home cage and was placed alone in a covered rotational chamber, a 8.5 in diameter $\times 5$ in. deep aluminum weighing basket affixed to the top of a phonograph turntable. The turntable was tilted to produce a plane of rotation $20 \mathrm{deg}$ from the horizontal. Rotation was delivered under two parametric conditions. Subjects of one group received 78-rpm exposures for six 1-min periods that were separated by 10 -sec deceleration intervals. Subjects of the second group were given 10 such intermittent $1-\mathrm{min}$ rotations. Subjects were then returned to their home cages and were provided access to food and water at $2100 \mathrm{~h}$. Standard two-bottle preference testing was initiated $48 \mathrm{~h}$ after rotation and was conducted for 15 days. During preference testing, each subject's daily access to separate bottles containing tap water or the saccharin solution was interrupted only by the brief period required for determination of bottle weights and refillings as necessary. Daily saccharin preference scores were computed for each subject to reflect the percentage to which ingestion of the saccharin contributed to total daily fluid intake. Subjects that displayed rejection or marginal acceptance of the saccharin solution during the CS exposure period were eliminated. Rejection and marginal acceptance were collectively defined as changes of less than $3 \mathrm{~g}$ in preexposure and postexposure weights of the bottles containing the CS solutions.

\section{RESULTS AND CONCLUSIONS}

Three subjects failed to satisfy the criterion of CS acceptance. The remaining strong and weak conditioners failed to differ with respect to the quantity of the CS solution that was consumed during the prerotational access period. Mean postrotation saccharin preference scores of strong and weak conditioners exposed to the two rotational conditions are displayed in Figure 1 and

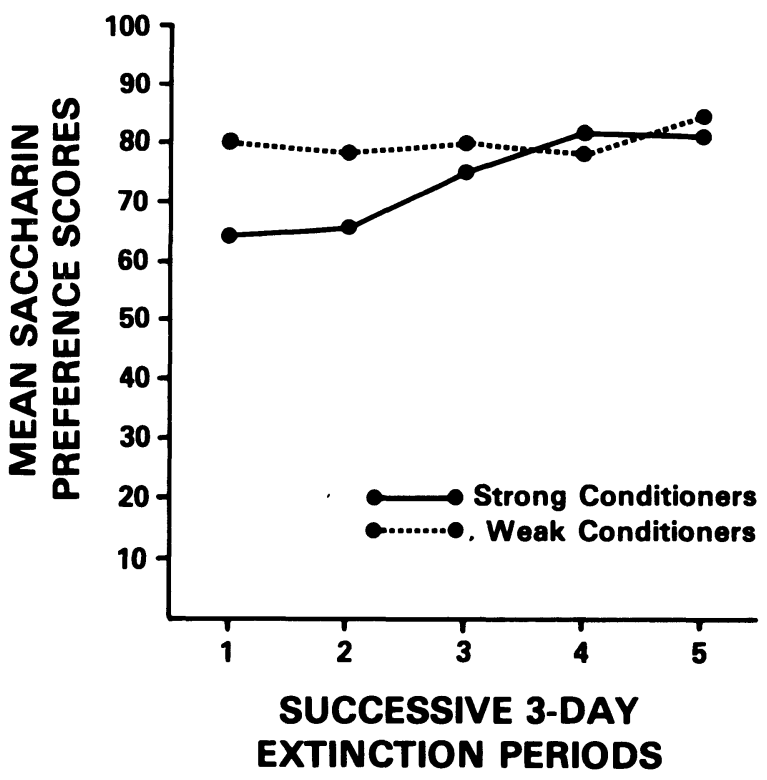

Figure 1. Mean successive 3-day saccharin preference scores of taste-aversion-prone and taste-aversion-resistant rats following a pairing of saccharin ingestion with $6 \mathrm{~min}$ of intermittent rotation. 


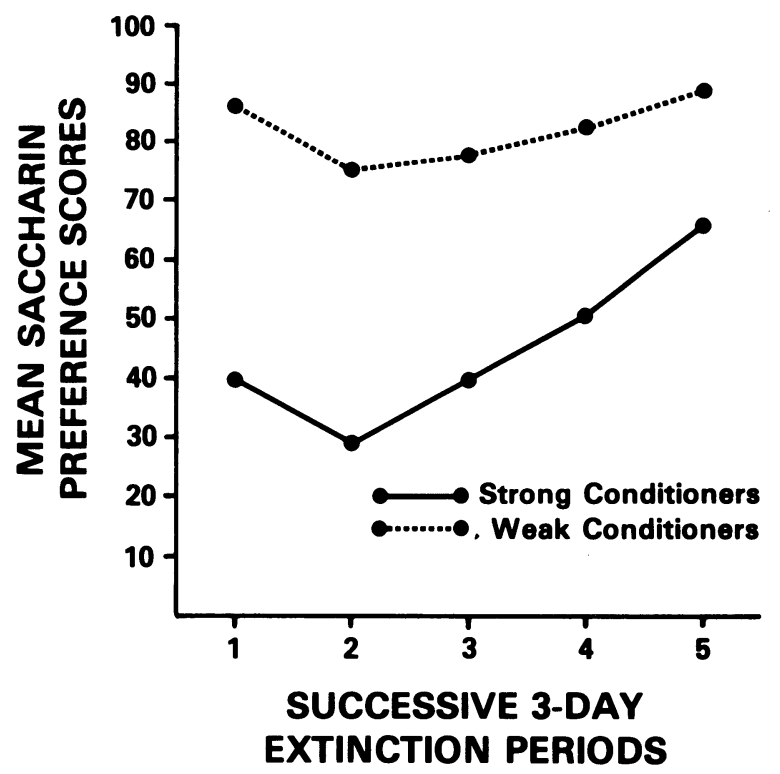

Figure 2. Mean successive 3-day saccharin preference scores of taste-aversion-prone and taste-aversion-resistant rats following a pairing of saccharin ingestion with $10 \mathrm{~min}$ of intermittent rotation.

Figure 2. Each subject's preference score was averaged across five successive 3-day extinction periods prior to graphical presentation or statistical analysis. Preliminary tests and inspection of the data provided no evidence of significant sex effects, so males and females were pooled for purposes of graphical presentation and additional analyses.

Visual inspection of the graphs suggests that rotation over 101 -min periods (Figure 2) had a much stronger effect than did rotation over six 1-min periods (Figure 1). These observations are statistically reliable. The 3-day averaged preference scores of each subject were subjected to an arcsin transformation as recommended by Winer (1962) prior to analysis of variance (ANOVA) tests. Data from the six 1-min rotations failed to produce a significant strain (between-subjects) effect $[F(1,17)=.32, p>.05]$, a significant effect for trials $[F(4,68)=2.47, p>.05]$, or a significant Strain by Trials interaction $[F(4,68)=1.59, \mathrm{p}>.1]$. This lack of significance is viewed as a probable "ceiling effect" indicative of insufficient illness induction for effective TA development. Mean saccharin preference scores of the weak conditioners are typical of those displayed by untreated controls in a variety of experiments, including a study of radiation-induced aversions to be discussed below (Peacock \& Fuller, Note 2).

Additional support for the ceiling effect interpretation and for the generality of strain effects across different methods of illness induction is provided by results of the longer rotational procedure. Analysis of data from the 101 -min rotations produced significant strain $[F(1,15)=6.38, p<.05]$ and trials $[F(4,60)=5.65$, $\mathrm{p}<.01$ ] effects, but a nonsignificant Strain by Trials interaction $[F(4,60)=1.66, p>.1]$. These results, in conjunction with Figure 2, support the conclusion that in contrast with the weak conditioners, the strong conditioners developed marked and relatively long-lasting aversions that were still apparent after 15 days of extinction testing.

These findings support the generality of strain effects across different illness-inducing procedures, thereby countering the argument that strain selection has been based on differential sensitivity to cyclophosphamide, the illness-inducing agent used in the process of genetic selection.

Some additional support for trans-US generality of strain effects has been provided by Peacock and Fuller (Note 2), who studied the acquisition of saccharin aversions in strong and weak conditioners while using ionizing X-radiation as the source of illness induction. Unlike the present study, which included two magnitudes of US exposure, Peacock and Fuller compared aversion formation of strong and weak TA learners with saccharin preferences of sham-exposed control subjects. Standard two-bottle preference tests reveal equivalent and high saccharin preference scores of approximately 90 for sham-irradiated subjects. The selected radiation dose, 50 roentgens, produced strong and initially equivalent aversions in both strong and weak conditioners. However, more rapid and more complete aversion extinction was observed in weak as opposed to strong conditioners. Resistance to extinction has been advanced as a useful indicator of relative aversion strength against a baseline of such "floor effects" (Elkins, 1973), and the Peacock and Fuller observation of accelerated extinction of weak conditioners is viewed as supporting the generality of strain effects. Additional radiation-based experiments will be conducted when resources permit. It is predicted that parametric manipulations of exposure doses across lower radiation doses will result in significant strain separation, as indicated by aversion formation by strong but not by weak conditioners.

\section{REFERENCE NOTES}

1. Elkins, R. L. Differential learning of illness-induced taste aversions and shock-motivated environmental avoidances by rats selectively bred for taste-aversion proneness or resistance to taste aversion acquisition. Paper presented at the annual meeting of the Southeastern Psychological Association, New Orleans, Louisiana, 1982.

2. Peacock, L. J., \& Fuller, O. W. Radiation-induced taste aversion in two strains of rats. Paper presented at the annual meeting of the Southern Society for Philosophy and Psychology, Fort Worth, Texas, 1982.

\section{REFERENCES}

Elxins, R. L. Individual differences in bait-shyness: Effects of drug dose and measurement technique. Psychological Record, 1973, 23, 349-358.

Elxins, R. L. Aversion therapy treatment of alcoholism: Chem- 
ical, electrical or verbal imaginary? International Journal of the Addictions, 1975, 10, 157-209.

Elxins, R. L. Covert sensitization treatment of alcoholism: Contributions of successful conditioning to subsequent abstinence maintenance. Addictive Behaviors, 1980, 5, 67-89. (a)

Elkins, R. L. A reconsideration of the relevance of recent animal studies for development of treatment procedures for alcoholics. Drug and Alcohol Dependence, 1980, 5, 101-113. (b)

Elxins, R. L., \& Hoв88, S. H. Forgetting, preconditioning CS familiarization and taste aversion learning: An animal experiment with implications for alcoholism treatment. Behavior Research and Therapy, 1979, 17, 567-573.

GreEN, L., \& RACHLIN, H. The effect of rotation on the learning of taste aversions. Bulletin of the Psychonomic Society, 1973, $1,137-138$.

Haroutunian, V., \& Riccio, D. C. Acquisition of rotation- induced taste aversions as a function of drinking-treatment delay. Physiological Psychology, 1975, 3, 273-277.

RILEY, A. L., \& Clarke, C. M. Conditioned taste aversions: A bibliography. In L. M. Barker, M. R. Best, \& M. Domjan (Eds.), Learning mechanisms in food selection. Waco, Tex: Baylor University Press, 1977.

VozgtuIN, W. L. Conditioned reflex therapy of chronic alcoholism. Ten years experience with the method. Rocky Mountain Medical Journal, 1947, 44, 807-812.

VoaEL, M. D. The relation of personality factors to GSR conditioning of alcoholics: An exploratory study. Canadian Journal of Psychotherapy, 1960, 14, 275-276.

WINER, B. J. Statistical principles in experimental design. New York: McGraw-Hill, 1962.

(Received for publication December 1, 1982.) 